\title{
Towards automated proofs of observational properties
}

\author{
Narjes Berregeb ${ }^{1}$ and Riadh Robbana ${ }^{2}$ and Ashish Tiwari ${ }^{3}$ \\ ${ }^{1}$ Laboratoire d'Informatique de Productique et de Parallélisme, Institut National des Sciences Appliquées et de Tech- \\ nologie, Tunis. \\ e-mail: narjes.robbana@insat.rnu.tn \\ ${ }^{2}$ Laboratoire d'Informatique de Productique et de Parallélisme, Ecole Polytechnique de Tunisie. \\ e-mail: riadh.robbana@fst.rnu.tn \\ ${ }^{3}$ SRI International. \\ e-mail: tiwari@csl.sri.com
}

\begin{abstract}
Observational theories are a generalization of first-order theories where two objects are observationally equal if they cannot be distinguished by experiments with observable results. Such experiments, called contexts, are usually infinite. Therefore, we consider a special finite set of contexts, called cover-contexts, "covering" all the observable contexts. Then, we show that to prove that two objects are observationally equal, it is sufficient to prove that they are equal (in the classical sense) under these cover-contexts. We give methods based on rewriting techniques, for constructing such cover-contexts for interesting classes of observational specifications.
\end{abstract}

Keywords: observational, contexts, rewriting

\section{Introduction}

A fundamental aim of formal specifications is to provide a rigorous basis to establish software correctness. Intuitively, a program is correct w.r.t its initial specification if it satisfies all the properties required by this specification. Behavioural abstraction provides a suitable basis for a more adequate notion of correctness. In a behavioural or observational theory, two objects are viewed as being identical if they cannot be ditinguished by observable experiments. Thus, for proving the correctness of a program, behavioural (or observational) concepts allow to abstract away from internal implementation details, and to focus only on its observable behaviour.

For instance, in the field of object-oriented programming, an observable experiment consists of an application of a method that returns certain visible attributes of that object. The actual implementation of the object is not crucial as long as the visible attributes returned by certain methods (and the iterative application of these methods) satisfy the program specification. When formalized using algebraic specifications, method names map to a sorted signature and the observable experiments map to contexts (terms over the signature with a "hole" for the invisible object) with visible sorts.

The idea that the semantics of a specification must describe the behaviour of an abstract datatype is due to [Gut75]. A lot of work has been devoted to the semantical aspects of observability and provability 
of observational equivalence, see for example [BBK94, Rei95. JR97, HB99. GM99]. In the framework of algebraic specifications, experiments with observable results are represented by particular terms called observable contexts. The main difficulty when dealing with proofs of observational properties is that the number of observable contexts is often infinite, and it had been shown in [Sch92] that there is no finite axiomatization of the behavioural equality in first-order logic.

In the framework of initial algebras, an algorithm was given in [BBR98] for constructing a finite set of contexts, that allows to describe the whole set of observable contexts. It applies to specifications represented by a left-linear system. We consider this construction in a more general framework (the semantic we use is not limited to only initial algebra), give more intuitions about it, and show that it can be used by any first order theorem prover for proving observational properties. We call the contexts obtained cover-contexts. Then, to prove that two terms are observationally equal, it is sufficient to prove that they are equal (in the classical sense) under these cover-contexts. We also show that the algorithm applies to an interesting class of non left-linear (equational) specifications with observable sorts. Finally, we consider the general case of non left-linear rewriting systems, we propose a procedure which outputs a cover-context set when it terminates.

\section{Related work}

Hennicker [Hen91] has proposed an induction principle, called context induction, which is a proof principle for behavioural abstractions. A context $c$ is viewed as a particular term containing exactly one variable; therefore, the subterm ordering defines a Noetherian relation on the set of observable contexts. Consequently, the principle of structural induction induces a proof principle for properties of contexts of observable sort, which is called context induction. This approach provides a uniform proof method for the verification of behavioural properties. It has been implemented in the system ISAR [BH93]. However in concrete examples, this verification is a non trivial task and requires human guidance: the system often needs a generalization of the current induction assertion before each nested context induction, so as to achieve the proof.

Malcolm and Goguen [GM00] suggested doing coinduction proofs by first defining a relation, showing it is a behavioural or hidden congruence, and then showing behavioural equivalence of two terms by showing that they are congruent. This technique, which they call "hidden coinduction," is easily automated only in certain cases where the specification satisfies additional strong restrictions. Note that checking if a relation is a hidden congruence involves establishing observational equivalence. Moreover, if the candidate relation is not a hidden congruence, then users have to find another candidate to complete the proof. The same problems appear in the approach of Bidoit and Hennicker [BH96] where users have to provide partial congruences.

Several other proof tools have been developed to aid coinductive proofs, but all of them require the user to supply an appropriate relation which the system can then prove to be a bisimulation. In the work of [DBG96], an automatic method is given to construct a bisimulation relation, however it uses heuristics and can fail on some examples.

In [MF98], an algorithm is proposed to generate the contextual equality, which coincides with the behavioural equivalence, by eliminating the redundant observational contexts using rewriting techniques. However, this algorithm applies to specifications with only one hidden sort. 


\section{Basic notions}

We assume that the reader is familiar with the basic concepts of algebraic specifications [Wir90], term rewriting and equational reasoning. A many sorted signature $\Sigma$ is a pair $(S, F)$ where $S$ is a set of sorts and $F$ is a set of function symbols. We assume a partition of $F$ into two subsets $C$ and $D$ of constructors symbols and defined function symbols. Let $X$ be a family of sorted variables and let $T(F, X)$ be the set of sorted terms. Let $\operatorname{var}(t)$ denote the set of variables appearing in $t$. A term is linear if all its variables occur only once. If $\operatorname{var}(t)$ is empty then $t$ is a ground term. The set of all ground terms is also denoted by $T(F)$. A term in $T(C, X)$ is called a constructor term. Let $A$ be an arbitrary non empty set, and let $F_{A}=\left\{f_{A} \mid f \in F\right\}$ such that if $f$ is of arity $n$, then $f_{A}$ is a function from $A^{n}$ to $A$. The pair $\left(A, F_{A}\right)$ is called a $\Sigma$-algebra, and $A$ is the carrier of the algebra. For sake of simplicity, we will write $A$ to denote the $\Sigma$-algebra when $F$ and $F_{A}$ are non ambiguous. A substitution $\eta$ assigns terms of appropriate sorts to variables. The domain of $\eta$ is denoted by $\operatorname{dom}(\eta)$. If $t$ is a term, then $t \eta$ denotes the application of $\eta$ to $t$. If $\eta$ replaces every variable by a ground term, then $\eta$ is a ground substitution. We denote by $\equiv$ the syntactic equivalence between objects.

Let $N^{*}$ be the set of finite sequences of positive integers. For any term $t, \operatorname{Pos}(t) \subseteq N^{*}$ denotes the set of positions of $t$, and the expression $t / u$ denotes the subterm of $t$ at position $u$. The root position is denoted by $\varepsilon$. The depth of a position $u$, denoted by $|u|$, is the length of the corresponding sequence. We denote by $t(u)$ the symbol of $t$ at position $u$. A position $u$ in a term $t$ is said to be a strict position if $t(u) \in F$. Let $u$ and $v$ two positions, if there exists a position $w$ such that $\mathrm{uw}=\mathrm{v}$ then $u \prec v$. We write $t[s]_{u}$ to indicate that $s$ is a subterm of $t$ at position $u$. We use also the notation $t\left[s_{1}, \ldots, s_{n}\right]$ to indicate that the term $t$ contains the subterms $s_{1}, \ldots, s_{n}$. The depth of a term $t$ is defined as follows: $|t|=0$ if $t$ is a constant or a variable, otherwise, $\left|f\left(t_{1}, \ldots, t_{n}\right)\right|=1+\max _{i}\left|t_{i}\right|$. An equation is a formula of the form $l=r$. It will be called a rewrite rule and written $l \rightarrow r$ if interest is in the left to right use of this equation. The term $l$ is the left-hand side of the rule. A rewrite rule $l \rightarrow r$ is left-linear if $l$ is linear. A rewrite system is a set of rewriting rules. Let $t$ be a term and $u$ a position in $t$. We write: $t \rightarrow_{R} s$ if there exists a rule $l \rightarrow r$ in $R$ and a substitution $\sigma$ such that $t / u=l \sigma, s / u=r \sigma$ and $t / v=s / v$ for any position $v$ such that $u \nprec v$. A term $t$ is irreducible (or in normal form) if there is no term $s$ such that $t \rightarrow_{R} s$. A rewrite system $R$ is said to be terminating if there is no infinite derivation $t_{1} \rightarrow_{R} t_{2} \rightarrow_{R} \cdots$ starting from any term $t_{1}$. If $\succ$ is a reduction ordering on terms such that $l \succ r$ for every $l \rightarrow r \in R$, then $R$ is terminating. A term $t$ is ground reducible if all its ground instances are reducible. A valid property $t_{1}=t_{2}$ in $R$, is denoted by $R \models t_{1}=t_{2}$. A theorem $t_{1}=t_{2}$ of $R$, is denoted by $R \vdash t_{1}=t_{2}$. An operator $f \in D$ is sufficiently complete iff for all $t_{1}, \ldots, t_{n} \in T(C)$, there exists $t \in T(C)$ such that $f\left(t_{1}, \ldots, t_{n}\right) \stackrel{*}{\rightarrow} t$. A rewriting system $R$ is sufficiently complete if each $f \in D$ is sufficiently complete.

\section{Observational Semantics}

The notion of observations has been introduced as a means for describing what is observed in a given algebra. Various techniques have been proposed: observations based on sorts, operators, terms or formula (see [BBK94] for a survey). The semantics we choose is based on a relaxing of the satisfaction relation. The notion of context is fundamental in all approaches based on such observational semantics. An observational property is obtained by taking into account only observable information. To show that it is valid, one has to show its validity in all observable contexts.

\footnotetext{
$\dagger$ A reduction ordering is a well-founded ordering that is closed under contexts and substitutions.
} 


specification: STACK
sorts: nat, stack
observable sorts: nat
constructors:
$0: \rightarrow$ nat
s: nat $\rightarrow$ nat
Nil: $\rightarrow$ stack
push: nat $\times$ stack $\rightarrow$ stack
defined operators
top: $\operatorname{stack} \rightarrow$ nat
pop: $\operatorname{stack} \rightarrow$ stack
axioms:
top $($ push $(x, y))=\mathrm{x}$
pop $($ push $(x, y))=y$

Fig. 1: Stack specification

Definition 1 (Context) Let $T(F, X)$ be a term algebra and $(S, F)$ be its signature.

- a context over $F$ is a non ground term $c \in T(F, X)$ with one distinguished occurrence of a variable called the contextual variable of $c$. To indicate the contextual variable $z_{s}$ occurring in $c$, we often write $c\left[z_{s}\right]$ instead of $c$, where $s$ is the sort of $z_{s}$.

- a context reduced to a variable $z_{s}$ of sort $s$ is called an empty context variable of sort s.

- the application of a context $c\left[z_{s}\right]$ to a term $t \in T(F, X)$ of sort $s$, denoted by $c[t]$, is defined by the substitution of $z_{s}$ by $t$ in $c\left[z_{s}\right]$. In this case, the context $c$ is said to be applicable to $t$.

- by assumption, $\operatorname{var}(c)$ will denote the set of variables occurring in c except the contextual variable of $c$. A context $c$ is ground if $\operatorname{var}(c)=\emptyset$. We denote by $|c|$ the depth of $c$.

- a subcontext (resp. strict subcontext) of c, is a context which is a subterm (resp. strict subterm) of $c$, having the same contextual variable as $c$.

Notations Let $c\left[z_{s}\right]$ and $c^{\prime}\left[z_{s^{\prime}}^{\prime}\right]$ be contexts such that $c^{\prime}$ is of sort $s$, let $t$ be a term and $\sigma$ be a substitution such that $z_{s} \notin \operatorname{dom}(\sigma)$. We use the following notations:

- $c\left[\left(c^{\prime}[t]\right)\right]=\left(c\left[c^{\prime}\right]\right)[t]=c\left[c^{\prime}[t]\right]$

- $(c[t]) \sigma=(c \sigma)[t \sigma]=c[t] \sigma$

Definition 2 (Specification, Observable specification) A specification (or equational specification) SP is a triple $(S, F, E)$ where $(S, F)$ is a signature and $E$ is a set of equations. An observational specification $S P_{o b s}$ is a couple $\left(S P, S_{\text {obs }}\right)$ such that $S P=(S, F, E)$ is a specification and $S_{o b s} \subseteq S$ is the set of observable sorts.

In the following we denote by $S P_{o b s}=\left(S P, S_{o b s}\right)$ an observational specification, where $S P=(S, F, E)$. We denote by $R$ the rewriting system associated with $S P$. 
Example 1 The Stack specification in Figure 1 is an observational specification where $S_{\text {obs }}=\{$ nat $\}$.

Definition 3 (Observable context) An observable context is a context whose sort belongs to $S_{\text {obs. }}$ The set of observable contexts is denoted by $C_{o b s}$.

Example 2 Consider the specification in Figure 1 there are infinitely many observable contexts:

- $\operatorname{top}\left(z_{\text {stack }}\right)$,

- $\operatorname{top}\left(\operatorname{pop}\left(z_{\text {stack }}\right)\right), \ldots$,

- $\operatorname{top}\left(\operatorname{pop}\left(\ldots\left(\operatorname{pop}\left(z_{\text {stack }}\right)\right) \ldots\right)\right), \ldots$,

- $\operatorname{top}\left(\operatorname{push}\left(i, z_{\text {stack }}\right)\right)$,

- $\operatorname{top}\left(\operatorname{push}\left(i, \operatorname{pop}\left(z_{\text {stack }}\right)\right)\right), \ldots$

The notion of observational validity is based on the idea that two objects are equal if they cannot be distinguished by observable contexts.

Definition 4 (Observational validity) Let $t_{1}, t_{2}$ be two terms. We say that $t_{1}=t_{2}$ is observationally valid, and denote it by $E \models{ }_{o b s} t_{1}=t_{2}$, iff for all ground $c_{o b s} \in C_{o b s}, E \models c_{o b s}\left[t_{1}\right]=c_{o b s}\left[t_{2}\right]$. We say that $t_{1}$ and $t_{2}$ are observationally equal and denote it by $t_{1}={ }_{o b s} t_{2}$, iff $E \models{ }_{o b s} t_{1}=t_{2}$.

Note that if $E \models t_{1}=t_{2}$ then $E \models{ }_{o b s} t_{1}=t_{2}$, but the converse may not be true. Observational theories generalize first-order theories: if $S_{o b s}=S$ then the satisfaction relation $\models_{o b s}$ is equal to $\models$.

Example 3 Consider the Stack specification in Figure 1. It is easy to see that push $($ top $(s), p o p(s))=s$ is not satisfied (in the classical sense), because push (top $(N i l), p o p(N i l))=N i l$ is not valid. However, it is observationally satisfied if we just observe the elements of the sequences push $($ top $(s), p o p(s))$ and $s$. This can be formally shown by considering all observable ground contexts.

\section{Cover-contexts}

The main problem of proving observational properties is that the number of observable ground contexts is often infinite. We introduce in this section the notion of cover-contexts which allow to describe finitely the often infinite set of observable ground contexts.

Definition 5 (Cover-tree) A cover-tree $T_{s}$ is a tree such that:

- The root is a contextual variable $z_{s}$ with $s \notin S_{o b s}$

- The successors of a node which is a non observable context $c\left[z_{s}\right]$ of sort $s_{i}$, are all the contexts of the form $f\left(x_{1}, \ldots, x_{i-1}, c, x_{i+1}, \ldots, x_{n}\right)$, where:

- $f \in F, f: s_{1} \times \ldots \times s_{i-1} \times s_{i} \times s_{i+1} \times \ldots \times s_{n} \rightarrow s^{\prime}$

- c is of sort $s_{i},\left(s_{i} \notin S_{\text {obs }}\right)$.

$-x_{1}, \ldots, x_{i-1}, x_{i+1}, \ldots, x_{n}$ are new variables not occuring in $c\left[z_{s}\right]$.

A path from a node $n_{1}$ to a node $n_{p}$ is a sequence of contexts $\left(n_{1}, n_{2}, \ldots, n_{p}\right)$ such that each $n_{i}$ is a successor of $n_{i-1}$, for $2 \leq i \leq p$. The depth of $T$, denoted by depth $(T)$ is the length of the longest path of $T$. 


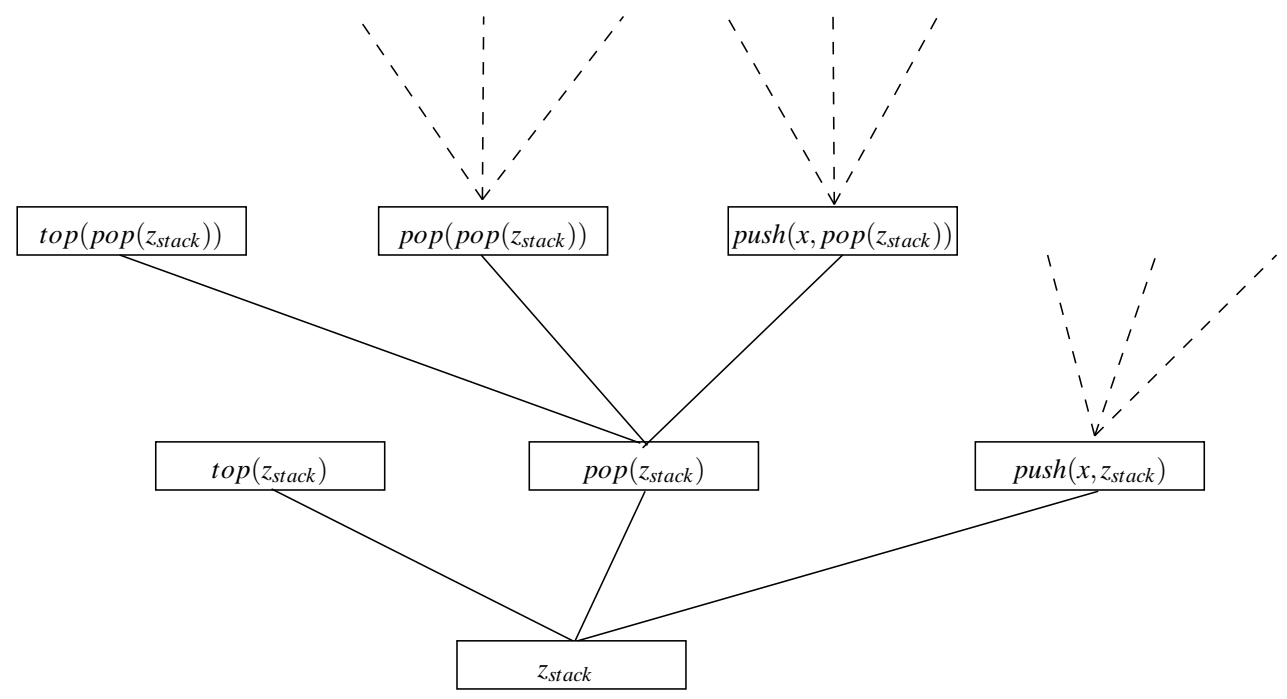

Fig. 2: A cover-tree for the stack specification

Example 4 A cover-tree $T_{\text {stack }}$ for the stack specification is given in Figure 2

Note that such cover-tree is often infinite. Each node is a context embedding its predecessor contexts in the same branch, and each leaf is an observable context. An interesting point is that, for proving that $a={ }_{o b s} b$ where $a$ and $b$ have a sort $s \notin S_{o b s}$, it is sufficient to consider a finite set of contexts $\left\{c_{1}, c_{2}, \ldots, c_{n}\right\}$ such that at least one context $c_{i}$ occurs in each path starting from the root $z_{s}$. In fact, if we have $c_{i}[a]=c_{i}[b]$ for each $c_{i}, 1 \leq i \leq n$, then we can deduce $c_{o b s}[a]=c_{o b s}[b]$ for each observable context $c_{o b s}$, since by construction of $T_{s}$, the contexts $c_{i}(1 \leq i \leq n)$ are subcontexts of all possible observable contexts. For example, if $t_{1}$ and $t_{2}$ are terms of sort stack, we can show if $t_{1}={ }_{o b s} t_{2}$ by considering only the set of contexts $\left\{\operatorname{top}\left(z_{\text {stack }}\right), \operatorname{pop}\left(z_{\text {stack }}\right), \operatorname{push}\left(x, z_{\text {stack }}\right)\right\}$. We can also consider the set $\left\{\operatorname{top}\left(z_{\text {stack }}\right), \operatorname{top}\left(\operatorname{pop}\left(z_{\text {stack }}\right)\right), \operatorname{pop}\left(\operatorname{pop}\left(z_{\text {stack }}\right)\right), \operatorname{push}\left(x, \operatorname{pop}\left(z_{\text {stack }}\right)\right), \operatorname{push}\left(x, z_{\text {stack }}\right)\right\}$. Note that there usually are infinitely many possibilities for choosing a set of contexts like $\left\{c_{1}, c_{2}, \ldots, c_{n}\right\}$. An interesting refinement is to consider in $T_{s}$, not the whole set of contexts $c\left[z_{s}\right]$, but only contexts that can be embedded in an observable ground irreducible context. We call such contexts cover-contexts. In other terms, instead of reasoning on the set of all observable contexts, we consider just an equivalent subset which is the set of ground observable and irreducible contexts.

Definition 6 (Quasi ground reducibility) A context c is quasi ground reducible if for all ground substitutions $\tau$ such that $\operatorname{dom}(\tau)=\operatorname{var}(c)$, c $\tau$ is reducible.

Example 5 The context top $\left(\right.$ push $\left.\left(x, z_{\text {stack }}\right)\right)$ is quasi ground reducible. But the context top $\left(z_{\text {stack }}\right)$ is not quasi ground reducible.

Definition 7 (Cover-context set) A cover-context set is a finite set of contexts $C C=\left\{c_{1}, c_{2}, \ldots, c_{n}\right\}$ such that:

i/ for each $c_{i} \in C C$, there exists an observable context $c_{o b s}$ such that $c_{o b s}\left[c_{i}\right]$ is not quasi ground reducible. 
ii/ for each ground irreducible observable context $c_{o b s}$, there exists $c_{i} \in C C, c_{o b s}^{\prime} \in C_{o b s}$ and a ground substitution $\tau$, such that $c_{o b s}=c_{o b s}^{\prime}\left[c_{i}\right] \tau$.

Property i expresses the minimality of $C C$ : only contexts that can be embedded in an observable ground irreducible context are retained. Property lii expresses the completeness of $C C$ : each ground observable context is "covered", in some sense, by a context $c$ of $C C$.

The following lemma shows that cover-contexts are sufficient for proving an observational property in first-order logic.

Lemma 1 Let $R$ be a terminating rewriting system such that $\operatorname{var}(r) \subseteq \operatorname{var}(l)$ for each rule $l \rightarrow r$ in $R$, and let $S_{\text {obs }}$ denotes the observable sorts.

Then, $R \models_{\text {obs }} t_{1}=t_{2}$ if $R \models c\left[t_{1}\right]=c\left[t_{2}\right]$ for all $c \in C C$.

Proof:

Let $c_{o b s}$ be an observable ground context. If $c_{o b s}$ is irreducible, then there exists a context $c \in C C$, a context $c_{o b s}^{\prime} \in C_{o b s}$ and a ground substitution $\tau$ such that $c_{o b s}=c_{o b s}^{\prime}[c] \tau$ (by second property of a covercontext set). We have $R \models c\left[t_{1}\right]=c\left[t_{2}\right]$ (by hypothesis), and therefore, $R \models\left(c_{o b s}^{\prime}[c] \tau\right)\left[t_{1}\right]=\left(c_{o b s}^{\prime}[c] \tau\right)\left[t_{2}\right]$. Thus, $R \models c_{o b s}\left[t_{1}\right]=c_{o b s}\left[t_{2}\right]$. If $c_{o b s}$ is reducible, then there exists an irreducible term $t$ such that $R \models$ $c_{o b s}[z]=t$ (since $R$ is terminating). There are three cases here:

(i) If the variable $z$ does not occur in $t$, then clearly $R \models c_{o b s}\left[t_{1}\right]=t$ and $R \models c_{o b s}\left[t_{2}\right]=t$, and hence $R \models c_{o b s}\left[t_{1}\right]=c_{o b s}\left[t_{2}\right]$.

(ii) If the variable $z$ occurs exactly once in $t$, then $t=c_{o b s}^{\prime}[z]$, where $c_{o b s}^{\prime}$ is an observable irreducible ground context. In this case, the argument given above shows that $R \models c_{o b s}^{\prime}\left[t_{1}\right]=c_{o b s}^{\prime}\left[t_{2}\right]$, and hence $R \models c_{o b s}\left[t_{1}\right]=c_{o b s}\left[t_{2}\right]$.

(iii) Finally, if the variable $z$ occurs more than once in $t$, then we consider the context $t^{1}$ obtained by replacing all but one occurrences of $z$ by $t_{1}$. Then we have: $c_{o b s}\left[t_{1}\right] \rightarrow_{R}^{*} t^{1}\left[t_{1}\right]$. If $t^{1}$ is reducible, we normalize it by $R$ to $t^{\prime}$ and if $z$ occurs more than once in $t^{\prime}$, we consider the term $t^{2}$ obtained by replacing all but one occurrences of $z$ by $t_{1}$. We repeat the process. If this process does not terminate, then we have an infinite derivation $c_{o b s}\left[t_{1}\right] \rightarrow_{R}^{*} t^{1}\left[t_{1}\right] \rightarrow_{R}^{*} t^{2}\left[t_{1}\right] \rightarrow_{R}^{*} \cdots$, which is impossible since $R$ is terminating. Therefore, the above process terminates with an irreducible term $u$ which contains at most one occurrence of $z$. Thus, this reduces to either case i or case iilabove.

\section{Computation of cover-context sets for left-linear systems}

Let us first introduce some useful definitions. We define $\operatorname{sdepth}(R)$ as the maximal depth of strict positions in left-hand sides of $R$. We define $\operatorname{depth}(R)$ as the maximal depth of positions in left-hand sides of $R$.

The idea of the computation is the following: for all non observable sorts $s$, we construct a cover-tree $T_{s}$ of depth equal to $\operatorname{sdepth}(R)$. Then we consider the set $L$ of all the leaves of all $T_{s}$. Starting from the non quasi ground reducible observable contexts of $L$, we add all contexts of $L$ that can be embedded in one of those observable contexts, to give a non quasi ground reducible and observable context. The algorithm is given in Figure 3 . This computation terminates since the trees $T_{s}$ are finite, for all $s \notin S_{\text {obs }}$. 
Since the depth of the cover-trees $T_{s}$ is sdepth $(R)$, the cover-contexts constructed will have a depth smaller than or equal to $\operatorname{sdepth}(R)$. However, we can reduce the number of cover-contexts by considering only subcontexts (for example of depth smaller than or equal to 1 ) of the constructed cover-contexts. Example 7 illustrates this idea.

The Ground reducibility is decidable for equational rewriting systems [Pla85]. The test of ground reducibility relies on a special finite set of terms called test set. A test set is defined as a finite set $T S(R)$ of irreducible terms, such that a term is ground reducible iff all its instances by substitutions in $\operatorname{TS}(R)$ are reducible. Several algorithms have been proposed for computing a test set, for left-linear rewrite systems (for example see [JK89. SF95]). For non left-linear systems, the computation is more complex than the left-linear case but applies to any rewrite system (for example see [KNZ86, Kou92, SSF95]). The constructed test set verifies some important properties (see [Kou92]):

1/ (finiteness): $T S(R)$ is a finite set.

2/ (minimality): For any term $t$ in $T S(R)$, there exists a ground substitution $\tau$ such that $t \tau$ is ground and irreducible.

3/ (completeness): For any ground irreducible term $s$, there exists a term $t$ in $T S(R)$ and a ground substitution $\tau$ such that $s=t \tau$.

4/ (transnormality): Every non ground term in $T S(R)$ has an infinite number of ground irreducible instances.

5/ (coverage): Any non ground term $t$ in $T S(R)$ is of depth equal or greater than $\operatorname{depth}(R)$.

In the case of a left-linear rewrite system, only conditions $1,2,3$ and 5 are necessary, besides condition 5 is simplified as follows: Any non ground term $t$ in $T S(R)$ is of depth equal to $\operatorname{sdepth}(R)$.

Test substitutions allow to instantiate variables of a context $c$ (or a term $t$ ) by elements of the test set whose variables are renamed in order to check the existence of a ground irreducible instance of the context $c$ (or the term $t$ ).

Definition 8 (Test substitution) A test substitution for a context c (resp. a term $t$ ) is a substitution that instantiates all the variables in var $(c)$ (resp. the variables in var(t)) by terms taken from the test set (whose variables have been renamed).

Test sets are used to test ground reduciblity, this property can be expressed as follows: Let $t$ be a term and $\sigma$ be a test substitution such that $t \sigma$ is irreducible, then there exists a ground substitution $\rho$ such that $t \sigma \rho$ is ground and irreducible. The proof of this property in the left-linear case is based on the fact that for all variable $x$ in $t,|x \sigma|=\operatorname{sdepth}(R)$, so, no subterm of $t \sigma \rho$ can match a left-hand-side of a rule in $R$. In the non left-linear case, the proof uses the transnormality property to build an irreducible instance $\rho$ such that $t \sigma \rho$ is ground and irreducible (see for example [Kou92]).

The Quasi ground reducibility is decidable for equational rewriting systems [KC86]. To test whether a context $c\left[z_{s}\right]$ is quasi ground reducible, we apply all test substitutions to $c\left[z_{s}\right]$ and show that they are reducible.

Lemma 2 A context $c\left[z_{s}\right]$ is quasi ground reducible iff for all test substitutions $\sigma$ such that $\operatorname{dom}(\sigma)=$ $\operatorname{var}(c), c\left[z_{s}\right] \sigma$ is reducible. 
for each non observable sort s, construct a cover-tree $T_{s}$ of depth $\operatorname{sdepth}(R)$.

let $L V$ be the set of leaves of all cover-trees $T_{S}$.

$L:=\bigcup_{c \in L V}$ expand $(c)$ where expand $(c)$ is $c$ if $c$ is observable, otherwise expand $(c)$ is obtained from $c$ by instantiating its variables (except the contextual variable) in all possible ways by terms, such that the new obtained contexts have all their variables at the same depth $\operatorname{sdepth}(R)$.

$C C_{0}:=\{c \in L \mid c$ is observable and not quasi ground reducible $\} \cup\left\{z_{s} \mid\right.$ s is observable $\}$

$L_{0}:=\{c \in L \mid c$ is not observable $\}$

\section{repeat}

$C C_{i+1}:=C C_{i} \cup\left\{c \in L_{i} \mid \exists c_{i} \in C C_{i}\right.$ such that $c_{i}[c]$ is not quasi ground reducible $\}$

$L_{i+1}:=L_{i} \backslash C C_{i+1}$

until $C C_{i+1}=C C_{i}$

output $C C_{i}$

Fig. 3: Computation of cover-contexts for a left-linear system

Proof:

$\Leftarrow$

Suppose for all test substitutions $\sigma, c\left[z_{s}\right] \sigma$ is reducible. By property of test sets, we deduce that for all ground substitutions $\tau$ such that for all $x \in \operatorname{var}(c), x \tau$ is ground and irreducible: there exists a ground substitution $\rho$ such that for all $x \in \operatorname{var}(c), x \sigma \rho$ is ground and irreducible. Then, $c\left[z_{s}\right] \sigma \rho$ is reducible. Thus, for all ground substitutions $\tau$ such that $\operatorname{dom}(\tau)=\operatorname{var}(c), c\left[z_{s}\right] \tau$ is reducible.

$\Rightarrow$

Suppose that there exists a test substitution $\sigma$ such that $c\left[z_{s}\right] \sigma$ is irreducible. We have to show that we can build a ground substitution $\rho$ such that $\forall x \in \operatorname{var}(c), x \sigma \rho$ is ground and irreducible, and $c\left[z_{s}\right] \sigma \rho$ is irreducible, and Thus, $c\left[z_{s}\right]$ is not quasi ground reducible. The proof uses the same arguments than for showing that test sets allow to test for ground reducibility. Let us detail the linear case:

From condition $2 /$ of test sets, there exists a ground substitution $\rho$ such that $\sigma \rho$ is ground and irreducible. Suppose that $c\left[z_{s}\right] \sigma \rho$ is reducible. Then there exists a strict position $u$, a rule $g \rightarrow d$ and a substitution $\theta$ such that $c\left[z_{s}\right] \sigma \rho / u=g \theta$. Note that the position $u$ necessarily occurs in $c\left[z_{s}\right]$ since $\sigma \rho$ is irreducible. Since $g$ is linear and for all $x$ in $c\left[z_{s}\right]|x \sigma|=\operatorname{sdepth}(R)$, we can build a substitution $\alpha$ such that for all variable $x$ occuring at a position $v$ of $g, x \alpha=c\left[z_{s}\right] \sigma / u v$. Then, $c\left[z_{s}\right] \sigma / u=g \alpha$. Thus, $c\left[z_{s}\right] \sigma$ is reducible, contradiction.

Theorem 1 Let $R$ be a left-linear rewriting system. Then, the set of contexts CC output by the computation described in Figure 3 is a cover-context set. 
Proof: We have to show that $C C$ is a cover-context set w.r.t Definition 7

i/ Let $c \in C C$. Then there exists $i$ such that $c \in C C_{i}$. Let us show that there exists an observable context $c_{o b s}$ such that $c_{o b s}[c]$ is not quasi ground reducible. The proof is by induction on $i$ :

$i=0:$ in this case $c \in C C_{0}$. We set $c_{o b s}=z_{s}$ where $s$ is the sort of $c$.

$i>0$ : there exists $c_{i-1} \in C C_{i-1}$ such that $c_{i-1}[c]$ is not quasi ground reducible. If $c_{i-1} \in C_{o b s}$, then we set $c_{o b s}=c_{i-1}$. Otherwise, $\left|c_{i-1}\right|=\operatorname{sdepth}(R)$. By induction hypothesis, there exists an observable context $c_{o b s}$ such that $c_{o b s}\left[c_{i-1}\right]$ is not quasi ground reducible. Let us show that $c_{o b s}\left[c_{i-1}[c]\right]$ is not quasi ground reducible.

$c_{o b s}\left[c_{i-1}\right]$ is not quasi ground reducible, therefore, there exists a test substitution $\sigma$ such that $\operatorname{dom}(\sigma)=$ $\operatorname{var}\left(c_{o b s}\left[c_{i-1}\right]\right)$ and $\left(c_{o b s}\left[c_{i-1}\right]\right) \sigma$ is irreducible (by Lemma 2). Let $\sigma_{1}$ be the restriction of $\sigma$ to $\operatorname{var}\left(c_{o b s}\right)$, then $\left(c_{o b s} \sigma_{1}\right)\left[c_{i-1}\right]$ is still irreducible.

$c_{i-1}[c]$ is not quasi ground reducible, therefore, there exists a test substitution $\sigma_{2}$ such that $\operatorname{dom}\left(\sigma_{2}\right)=$ $\operatorname{var}\left(c_{i-1}\right) \cup \operatorname{var}(c)$ and $c_{i-1}[c] \sigma_{2}$ is irreducible (by Lemma 2 .

Let us show that $c_{o b s}\left[c_{i-1}[c]\right] \sigma_{1} \sigma_{2}$ is irreducible. We can then deduce, by property of test sets, a ground substitution $\tau$ such that $c_{o b s}\left[c_{i-1}[c]\right] \sigma_{1} \sigma_{2} \tau$ is ground and irreducible.

Suppose that $c_{o b s}\left[c_{i-1}[c]\right] \sigma_{1} \sigma_{2}$ is reducible, then there exists a rule $g \rightarrow d$, a substitution $\theta$ and a position $u$ such that $c_{o b s}\left[c_{i-1}[c]\right] \sigma_{1} \sigma_{2} / u=g \theta$. The position $u$ cannot occur in $c_{i-1}$, otherwise $c_{i-1}[c] \sigma_{2}$ would be reducible. Then, necessarily, $u$ occurs in $c_{o b s}$. Since $g$ is linear, we can build a substitution $\alpha$ such that for each variable $x$ appearing at a position $w$ of $g, x \alpha=\left(c_{o b s} \sigma_{1}\right)\left[c_{i-1}\right] / u w$. Then $\left(c_{o b s} \sigma_{1}\right)\left[c_{i-1}\right] / u=g \alpha$, which contradicts the fact that $\left(c_{o b s} \sigma_{1}\right)\left[c_{i-1}\right]$ is irreducible

ii/ Suppose that there exists an observable ground irreducible context $c_{o}$ such that there does not exist $c \in C C$ and a ground substitution $\tau$ such that $c \tau$ is a subcontext of $c_{o}$. Let us first define the top of a term $t$ as follows:

- $\operatorname{top}(t, d)=t$, if $|t| \leq d$

- $\operatorname{top}\left(f\left(t_{1}, \ldots, t_{n}\right), 0\right)=f\left(x_{1}, \ldots, x_{n}\right)$, where $x_{i}(i \in[1 . . n])$ are fresh variables.

- $\operatorname{top}\left(f\left(t_{1}, \ldots, t_{n}\right), d\right)=f\left(\operatorname{top}\left(t_{1}, d-1\right), \ldots\right.$, top $\left.\left(t_{n}, d-1\right)\right)$ otherwise.

Let $d$ be the depth of the position of the contextual variable of $c_{o}$. Let us choose $c_{o}$ such that $d$ is minimal. Let $c_{o b s}=\operatorname{top}\left(c_{o}, d\right)$. If $d \leq \operatorname{sdepth}(R)$ then $c_{o b s} \in C C$, contradiction. Otherwise, let $c$ be a subcontext of $c_{o b s}$ of depth $\operatorname{sdepth}(R)$, and let $c_{o b s}^{\prime}$ be an observable context such that $c_{o b s}=c_{o b s}^{\prime}[c]$. Note that $c_{o b s}^{\prime}[c]$ is not quasi ground reducible. By hypothesis, $c \notin C C$. Let $d^{\prime}$ be the depth of the contextual variable of $c_{o b s}^{\prime}$. We have, $d^{\prime}<d$, necessarily there exists $c^{\prime} \in C C, \tau^{\prime}, c_{o b s}^{\prime \prime}$ such that $c_{o b s}^{\prime}=c_{o b s}^{\prime \prime}\left[c^{\prime}\right] \tau^{\prime}$. In this case, $c^{\prime} \in C C$ since $c^{\prime}[c]$ is not quasi ground reducible, contradiction.

Example 6 Consider the specification in Figure 1 We have: $\operatorname{sdepth}(R)=1$. A test set for $R$ is:

$$
\{0, s(x), \operatorname{Nil}, \operatorname{push}(x, y)\} \text {. }
$$


Applying the computation principle described in Figure 3, we get:

$$
\begin{aligned}
C C_{0} & =\left\{z_{\text {nat }}, \operatorname{top}\left(z_{\text {stack }}\right)\right\} \\
L_{0} & =\left\{\operatorname{pop}\left(z_{\text {stack }}\right), \operatorname{push}\left(i, z_{\text {stack }}\right)\right\}
\end{aligned}
$$

In the next iteration, we add the context pop $\left(z_{\text {stack }}\right)$ since top $\left(p o p\left(z_{\text {stack }}\right)\right)$ is ground and irreducible.

$$
\begin{aligned}
C C_{1} & =\left\{z_{\text {nat }}, \text { top }\left(z_{\text {stack }}\right)\right\} \cup\left\{\text { pop }\left(z_{\text {stack }}\right)\right\} \\
L_{1} & =\left\{\text { push }\left(i, z_{\text {stack }}\right)\right\}
\end{aligned}
$$

$C C_{2}=C C_{1}$ is a cover-context set for $R$.

Definition 9 Let $f \in D$. We say that $f$ is strongly complete if $f\left(t_{1}, \ldots, t_{n}\right) \stackrel{*}{\rightarrow} t$ and $t \in T(C, X)$, for all $t_{i}(i \in[1 . . n]) \in(T(C, X) \backslash X)$. A rewriting system $R$ is strongly complete if for all $f \in D, f$ is strongly complete.

Note that: if $f$ is strongly complete, then $f$ is sufficiently complete. The converse may not be true. The following theorem states that we can compute a cover-context based on the algorithm given in Figure 3 . for a non left-linear rewriting systems provided that it is strongly complete and that the relations between defined functions are left-linear.

Theorem 2 Let $R$ be a rewriting system strongly complete, such that relations between defined functions are left-linear. We also assume that if a constructor cons $: s_{1} \times s_{2} \ldots s_{n} \rightarrow s$ is such that if $s$ is observable then $s_{1}, s_{2} \ldots s_{n}$ are also observable. Then, the set of contexts CC output by the computation described in Figure 3 is a cover-context set.

Proof:

i/ Let $c \in C C$. Then there exists $i$ such that $c \in C C_{i}$. Let us show that there exists an observable context $c_{o b s}$ such that $c_{o b s}[c]$ is not quasi ground reducible. The proof is by induction on $i$ :

$i=0$ : in this case $c \in C C_{0}$. We set $c_{o b s}=z_{s}$ where $s$ is the sort of $c$.

$i>0$ : there exists $c_{i-1} \in C C_{i-1}$ such that $c_{i-1}[c]$ is not quasi ground reducible. If $c_{i-1} \in C_{o b s}$, then we set $c_{o b s}=c_{i-1}$. Otherwise, $\left|c_{i-1}\right|=\operatorname{sdepth}(R)$. By induction hypothesis, there exists an observable context $c_{o b s}$ such that $c_{o b s}\left[c_{i-1}\right]$ is not quasi ground reducible. Thanks to the assumptions on constructors, we can choose $c_{o b s}$ such that $c_{o b s}(\varepsilon) \in D$ or $c_{o b s}(\varepsilon) \in X$. If $c_{o b s}(\varepsilon) \in X$ (empty context), then we have $c_{o b s}\left[c_{i-1}[c]\right]$ not quasi ground reducible. Otherwise, we have $c_{o b s}(\varepsilon) \in D$. Let us show then, that $c_{o b s}\left[c_{i-1}[c]\right]$ is not quasi ground reducible.

$c_{o b s}\left[c_{i-1}\right]$ is not quasi ground reducible, therefore, there exists a test substitution $\sigma_{1}$ such that $\operatorname{dom}\left(\sigma_{1}\right)=\operatorname{var}\left(c_{o b s}\right)$ and $\left(c_{o b s} \sigma_{1}\right)\left[c_{i-1}\right]$ is irreducible.

$c_{i-1}[c]$ is not quasi ground reducible, therefore, there exists a test substitution $\sigma_{2}$ such that $\operatorname{dom}\left(\sigma_{2}\right)=$ $\operatorname{var}\left(c_{i-1}\right) \cup \operatorname{var}(c)$ and $c_{i-1}[c] \sigma_{2}$ is irreducible.

Consider all subcontexts $f\left(t_{1}, \ldots, t_{k-1}, c^{\prime}\left[z_{s}\right], t_{k+1}, \ldots, t_{n}\right)$ of $c_{o b s}\left[c_{i-1}\right] \sigma_{1} \sigma_{2}$, where $t_{j}$ is a term for all $j \in\{1, \ldots, k-1, k+1, \ldots, n\}$, and $c^{\prime}$ is a context. Since $\left(c_{o b s} \sigma_{1}\right)\left[c_{i-1}\right]$ is irreducible and $R$ is strongly complete, then $t_{j} \in T(C, X)$. Besides $c_{o b s}(\varepsilon) \in D$. 


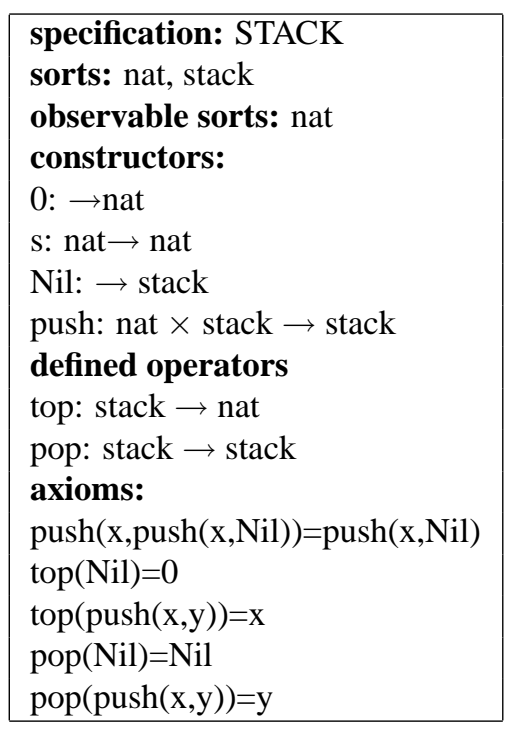

Fig. 4: A non left-linear specification

Let $u_{z}$ be the position of $z_{s}$ in $\left(c_{o b s} \sigma_{1}\right)\left[c_{i-1}\left[z_{s}\right]\right]$, and $u$ be a position in $\left(c_{o b s} \sigma_{1}\right)\left[c_{i-1}\left[z_{s}\right]\right]$ such that $u \prec u_{z}$. Necessarily, $\left(c_{o b s} \sigma_{1}\right)\left[c_{i-1}\right](u) \in D$, otherwise $\left(c_{o b s} \sigma_{1}\right)\left[c_{i-1}\right]$ would be reducible since $R$ is strongly complete.

Now, let us show that $c_{o b s}\left[c_{i-1}[c]\right] \sigma_{1} \sigma_{2}$ is irreducible. We can then deduce, by property of test sets, a ground substitution $\tau$ such that $c_{o b s}\left[c_{i-1}[c]\right] \sigma_{1} \sigma_{2} \tau$ is ground and irreducible. Suppose that $c_{o b s}\left[c_{i-1}[c]\right] \sigma_{1} \sigma_{2}$ is reducible, then there exists a rule $g \rightarrow d$, a substitution $\theta$ and a position $u$ such that $c_{o b s}\left[c_{i-1}[c]\right] \sigma_{1} \sigma_{2} / u=g \theta$. The position $u$ cannot occur in $c_{i-1}$, otherwise $c_{i-1}[c] \sigma_{2}$ would be reducible. Then, necessarily, $u$ occurs in $c_{o b s}$.

$u \nprec u_{z}$, then $\left(c_{o b s} \sigma_{1}\right)\left[c_{i-1}\right]$ would be reducible. Contradiction.

$u \prec u_{z}$ then $c_{o b s}(u) \in D$. In this case, $g$ is linear since the relations between defined symbols are left-linear. Then, we can build a substitution $\alpha$ such that for each variable $x$ appearing at a position $w$ of $g, x \alpha=\left(c_{o b s} \sigma_{1}\right)\left[c_{i-1}\right] / u w$. Then $\left(c_{o b s} \sigma_{1}\right)\left[c_{i-1}\right] / u w=g \alpha$, which contradicts the fact that $\left(c_{o b s} \sigma_{1}\right)\left[c_{i-1}\right]$ is irreducible.

ii/ The proof of the second property of cover-contexts is similar to the case where $R$ is left-linear.

Example 7 Consider an example of a non left-linear rewriting system given in Figure 4 The rewriting system is strongly complete and the relations between defined operators (top and pop) are left-linear. We have $\operatorname{sdepth}(R)=2$. A test set for $R$ is:

$$
\{0, s(0), s(s(x)), \operatorname{Nil}, \operatorname{push}(0, N i l), \operatorname{push}(s(x), N i l), \operatorname{push}(0, \operatorname{push}(x, y)), \operatorname{push}(s(x), \operatorname{push}(x, y))\}
$$


Applying the computation principle described in Figure 3 we get:

$$
\begin{aligned}
C C_{0}= & \left\{z_{\text {nat }}, \operatorname{top}\left(z_{\text {stack }}\right), \operatorname{top}\left(\operatorname{pop}\left(z_{\text {stack }}\right)\right)\right\} \\
L_{0}= & \left\{\operatorname{pop}\left(\operatorname{pop}\left(z_{\text {stack }}\right)\right), \operatorname{push}\left(0, \operatorname{pop}\left(z_{\text {stack }}\right), \operatorname{push}\left(s(x), \operatorname{pop}\left(z_{\text {stack }}\right),\right.\right.\right. \\
& \operatorname{push}(0, \operatorname{push}(x, z)), \operatorname{push}(s(y), \operatorname{push}(x, z))\}
\end{aligned}
$$

In the next iteration, we add the context pop $\left(\right.$ pop $\left.\left(z_{\text {stack }}\right)\right)$ since top $\left(\operatorname{pop}\left(\operatorname{pop}\left(z_{\text {stack }}\right)\right)\right)$ is ground and irreducible.

$$
\begin{aligned}
C C_{1}= & \left\{z_{\text {nat }}, \operatorname{top}\left(z_{\text {stack }}\right), \operatorname{top}\left(\operatorname{pop}\left(z_{\text {stack }}\right)\right)\right\} \cup\left\{\operatorname{pop}\left(\operatorname{pop}\left(z_{\text {stack }}\right)\right)\right\} \\
L_{1}= & \left\{\operatorname { p u s h } \left(0, \operatorname{pop}\left(z_{\text {stack }}\right), \operatorname{push}\left(s(x), \operatorname{pop}\left(z_{\text {stack }}\right), \operatorname{push}(0, \operatorname{push}(x, z)),\right.\right.\right. \\
& \operatorname{push}(s(y), \operatorname{push}(x, z))\}
\end{aligned}
$$

$C C_{2}=C C_{1}$ is a cover-context set for $R$.

We can refine $C_{1}$ by considering only subcontexts of depth smaller than or equal to 1 . This leads to the cover-context set $\left\{z_{\text {nat }}\right.$, top $\left(z_{\text {stack }}\right)$, pop $\left.\left(z_{\text {stack }}\right)\right\}$.

\section{Computation of cover-context sets for non left-linear systems}

The algorithm for computing a cover-context set for left-linear rewriting systems does not work for the non-linear case. For example, consider the following rewrite system $R$ :

$$
\begin{aligned}
g(g(x)) & \rightarrow x \\
f(g(x), x) & \rightarrow x \\
f(x, g(x)) & \rightarrow x \\
f(x, x) & \rightarrow x \\
f(x, f(x, z)) & \rightarrow x \\
f(g(x), f(x, z)) & \rightarrow x
\end{aligned}
$$

A test set for $R$ is $T S(R)=\{a, g(a)\}$. Consider the contexts $c_{1}=f\left(x_{1}, z\right), c_{2}=f\left(x_{2}, z\right), c_{3}=f\left(x_{3}, z\right)$. The context $c_{1}\left[c_{2}\right]$ has an irreducible ground instance which is $f(a, f(g(a), z))$. The context $c_{2}\left[c_{3}\right]$ has also an irreducible ground instance which is $f(a, f(g(a), z))$. However, the context

$$
c_{1}\left[c_{2}\left[c_{3}\right]\right]=f\left(x_{1}, f\left(x_{2}, f\left(x_{3}, z\right)\right)\right)
$$

has not an irreducible ground instance. Therefore, the cover-contexts computation for left-linear systems (see Figure 3), does not hold for non left-linear systems, since it is based on the idea that: if there exists $c_{i} \in C C_{i}$ such that $c_{i}[c]$ is a non quasi ground reducible context, then $c$ can be embedded in an observable ground irreducible context. For non left-linear systems, we use a stronger condition; we show that if there exists $c_{i} \in C C_{i}$ such that $c_{i}[c]$ has an infinite number of irreducible instances, then $c$ can be embedded in an observable ground irreducible context. We present in this section a procedure for computing a cover-context set for non left-linear rewriting systems, but which can diverge in some cases. Let us first introduce some useful definitions. 


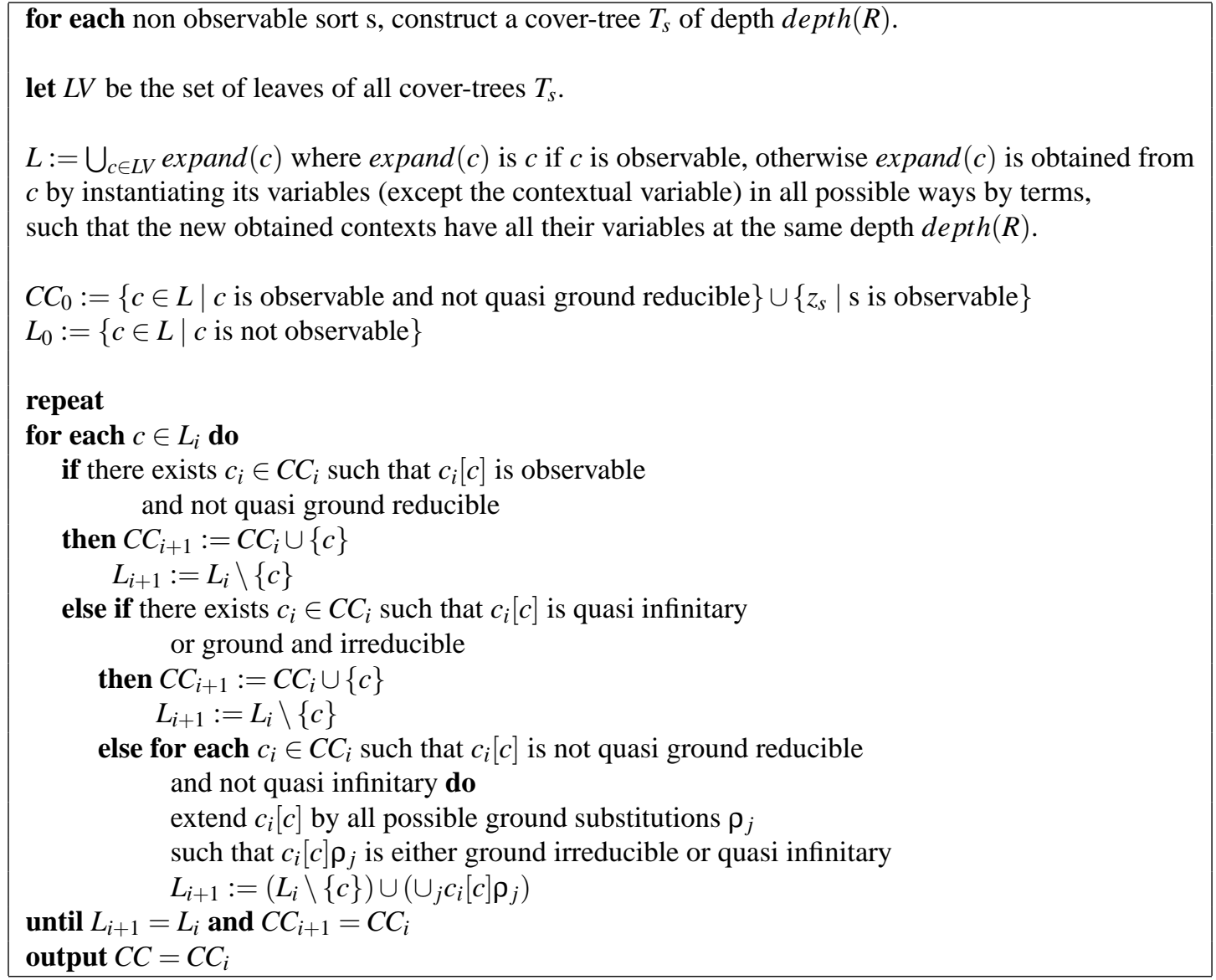

Fig. 5: Computation of cover-contexts for a non left-linear system

Definition 10 (Quasi infinitary) A context $c\left[z_{s}\right]$ is quasi infinitary iff there exists a test substitution $\sigma$ such that $\operatorname{dom}(\sigma)=\operatorname{var}(c)$, for all $x \in \operatorname{dom}(\sigma), x \sigma$ is not ground, and $c\left[z_{s}\right] \sigma$ is irreducible.

If $c$ is a quasi infinitary context, then $c$ has an infinite number of irreducible instances, thanks to the test set properties (transnormality).

Example 8 Consider the specification in Figure 4 The context push $\left(x, z_{s}\right)$ is quasi-infinitary since push $(s(y), z)$ is irreducible for all $y$.

Theorem 3 Let $R$ be a non left-linear rewriting system. Then, if the computation described in Figure 5 terminates, the set of contexts CC output is a cover-context set.

Proof: We have to show that $C C$ is a cover-context set w.r.t Definition 7

Let $c \in C C$. Then there exists $i$ such that $c \in C C_{i}$. Let us show that there exists an observable context $c_{o b s}$ such that $c_{o b s}[c]$ is not quasi ground reducible. The proof is by induction on $i$ : 
$i=0$ : in this case $c \in C C_{0}$. We set $c_{o b s}=z_{s}$ where $s$ is the sort of $c$.

$i>0$ : there exists $c_{i-1} \in C C_{i-1}$ such that $c_{i-1}[c]$ is not quasi ground reducible. If $c_{i-1} \in C_{o b s}$, then we set $c_{o b s}=c_{i-1}$. Otherwise, $\left|c_{i-1}\right|=\operatorname{depth}(R)$. By induction hypothesis, there exists an observable context $c_{o b s}$ such that $c_{o b s}\left[c_{i-1}\right]$ is quasi infinitary or ground irreducible. Let us show that $c_{o b s}\left[c_{i-1}[c]\right]$ is not quasi ground reducible.

$c_{o b s}\left[c_{i-1}\right]$ is not quasi ground reducible, therefore, there exists a ground substitution $\tau_{1}$ such that $\operatorname{dom}\left(\tau_{1}\right)=$ $\operatorname{var}\left(c_{o b s}\right)$ and $\left(c_{o b s} \tau_{1}\right)\left[c_{i-1}\right]$ is irreducible.

Suppose $c_{i-1}[c]$ is quasi infinitary (the proof of the case where it is ground and irreducible is a subcase), therefore, there exists a ground substitution $\tau_{2}$ such that $\operatorname{dom}\left(\tau_{2}\right)=\operatorname{var}\left(c_{i-1}\right) \cup \operatorname{var}(c), c_{i-1}[c] \tau_{2}$ is irreducible and for all $x, y \in \operatorname{var}\left(c_{i-1}[c]\right)$ :

$$
\begin{aligned}
\left|x \tau_{2}\right| & >\left|c_{o b s}\left[c_{i-1}\right] \tau_{1}\right| \\
|| x \tau_{2}|-| y \tau_{2}|| & >\left|c_{o b s}\left[c_{i-1}\right] \tau_{1}\right|
\end{aligned}
$$

Let us show that $c_{o b s}\left[c_{i-1}[c]\right] \tau_{1} \tau_{2}$ is irreducible.

Suppose that $c_{o b s}\left[c_{i-1}[c]\right] \tau_{1} \tau_{2}$ is reducible, then there exists a rule $g \rightarrow d$, a substitution $\theta$ and a position $u$ such that $c_{o b s}\left[c_{i-1}[c]\right] \tau_{1} \tau_{2} / u=g \theta$. The position $u$ cannot occur in $c_{i-1}$, otherwise $c_{i-1}[c] \tau_{2}$ would be reducible. Then, necessarily, $u$ occurs in $c_{o b s}$.

- If $g$ is linear, we can build a substitution $\alpha$ such that for each variable $x$ appearing at a position $w$ of $g$, $x \alpha=\left(c_{o b s} \tau_{1}\right)\left[c_{i-1}\right] / u w$. Then $\left(c_{o b s} \tau_{1}\right)\left[c_{i-1}\right] / u w=g \alpha$, which contradicts the fact that $\left(c_{o b s} \tau_{1}\right)\left[c_{i-1}\right]$ is irreducible.

- If $g$ is not linear, suppose there exists two positions $u_{1}$ and $u_{2}$ corresponding to a variable $x$ in $g$ such that

$$
c_{o b s}\left[c_{i-1}\right] \tau_{1} / u u_{1} \neq c_{o b s}\left[c_{i-1}\right] \tau_{1} / u u_{2}
$$

but

$$
c_{o b s}\left[c_{i-1}[c]\right] \tau_{1} \tau_{2} / u u_{1}=c_{o b s}\left[c_{i-1}[c]\right] \tau_{1} \tau_{2} / u u_{2}
$$

Let $u_{z}$ be the postion of the contextual variable of $c_{i-1}$ in $c_{o b s}\left[c_{i-1}\right]$.

case 1: $u_{1}$ occurs in $c_{o b s} \tau_{1} . u_{2}$ occurs in $c_{i-1}$.

case 1.1: $u_{2} \prec u_{z}$ and $u_{1} \nprec u_{z}$. In this case: $c_{o b s}\left[c_{i-1}[c]\right] \tau_{1} \tau_{2} / u u_{2}$ is not ground, but

$$
c_{o b s}\left[c_{i-1}[c]\right] \tau_{1} \tau_{2} / u u_{1}
$$

is ground, contradiction.

case 1.2: $u_{2} \prec u_{z}$ and $u_{1} \prec u_{z}$. In this case:

$$
\left|c_{o b s}\left[c_{i-1}[c]\right] \tau_{1} \tau_{2} / u u_{1}\right| \neq\left|c_{o b s}\left[c_{i-1}[c]\right] \tau_{1} \tau_{2} / u u_{1}\right|,
$$

contradiction.

case 1.3: $u_{1} \nprec u_{z}$ and $u_{2} \nprec u_{z}$. 
- if $c_{o b s}\left[c_{i-1}\right] / u u_{2}$ is ground, then $c_{o b s}\left[c_{i-1}[c]\right] \tau_{1} \tau_{2} / u u_{2}=c_{o b s}\left[c_{i-1}\right] \tau_{1} / u u_{2}$.

Besides:

$$
c_{o b s}\left[c_{i-1}[c]\right] \tau_{1} \tau_{2} / u u_{2}=c_{o b s}\left[c_{i-1}[c]\right] \tau_{1} \tau_{2} / u u_{1} .
$$

Therefore

$$
c_{o b s}\left[c_{i-1}\right] \tau_{1} / u u_{2}=c_{o b s}\left[c_{i-1}[c]\right] \tau_{1} \tau_{2} / u u_{1}=c_{o b s}\left[c_{i-1}\right] \tau_{1} / u u_{1},
$$

contradiction.

- if $c_{o b s}\left[c_{i-1}\right] / u u_{2}$ is not ground. Let $x$ be a variable occuring in $c_{o b s}\left[c_{i-1}\right] / u u_{2}$. We have:

$$
\left|x \tau_{2}\right|>\left|c_{o b s}\left[c_{i-1}\right] \tau_{1}\right|
$$

Therefore:

$\left|c_{o b s}\left[c_{i-1}[c]\right] \tau_{1} \tau_{2} / u u_{1}\right|=\left|c_{o b s}\left[c_{i-1}\right] \tau_{1} / u u_{1}\right|<\left|c_{o b s}\left[c_{i-1}\right] \tau_{1} \tau_{2} / u u_{2}\right|=\left|c_{o b s}\left[c_{i-1}\right] \tau_{1} \tau_{2} / u u_{1}\right|$, contradiction.

case 2: $u_{1}$ occurs in $c_{i-1} . u_{2}$ occurs in $c_{i-1}$.

case 2.1: $u_{2} \prec u_{z}$ and $u_{1} \nprec u_{z}$ In this case $c_{o b s}\left[c_{i-1}[c]\right] \tau_{1} \tau_{2} / u u_{2}$ is not ground and $c_{o b s}\left[c_{i-1}[c]\right] \tau_{1} \tau_{2} / u u_{1}$ is ground, contradiction.

case 2.2: $u_{2} \prec u_{z}$ and $u_{1} \prec u_{z}$. Similar to case 1.2

case 2.3: $u_{2} \nprec u_{z}$ and $u_{1} \nprec u_{z}$.

- if $c_{o b s}\left[c_{i-1}\right] / u u_{1}$ is ground and $c_{o b s}\left[c_{i-1}\right] / u u_{2}$ is ground. In this case

$$
c_{o b s}\left[c_{i-1}[c]\right] \tau_{1} \tau_{2} / u u_{1}=c_{o b s}\left[c_{i-1}\right] \tau_{1} / u u_{1}
$$

and

$$
c_{o b s}\left[c_{i-1}[c]\right] \tau_{1} \tau_{2} / u u_{2}=c_{o b s}\left[c_{i-1}\right] \tau_{1} / u u_{2}
$$

Therefore:

$$
c_{o b s}\left[c_{i-1}\right] \tau_{1} / u u_{1}=c_{o b s}\left[c_{i-1}\right] \tau_{1} / u u_{2}
$$

contradiction

- if $c_{o b s}\left[c_{i-1}\right] / u u_{1}$ is ground and $c_{o b s}\left[c_{i-1}\right] / u u_{2}$ is not ground. Let $x$ be a variable occuring in $c_{o b s}\left[c_{i-1}\right] / u u_{2}$. We have

$$
\left|x \tau_{2}\right|>\left|c_{o b s}\left[c_{i-1}\right] \tau_{1}\right| \geq\left|c_{o b s}\left[c_{i-1}\right] \tau_{1} / u u_{1}\right| .
$$

Therefore:

$$
\left|c_{o b s}\left[c_{i-1}[c]\right] \tau_{1} \tau_{2} / u u_{2}\right|>\left|c_{o b s}\left[c_{i-1}[c]\right] \tau_{1} \tau_{2} / u u_{1}\right|,
$$

contradiction. 
- if $c_{o b s}\left[c_{i-1}\right] / u u_{1}$ is not ground and $c_{o b s}\left[c_{i-1}\right] / u u_{2}$ is not ground. Let $x$ be a variable in $c_{o b s}\left[c_{i-1}\right] / u u_{1}$ such that

$$
\left|x \tau_{1}\right|=\max _{x_{i} \in c_{o b s}\left[c_{i-1}\right] / u u_{1}}\left|x_{i} \tau_{2}\right| .
$$

Let $y$ be a variable in $c_{o b s}\left[c_{i-1}\right] / u u_{2}$ such that

$$
\left|y \tau_{2}\right|=\max _{y_{i} \in c_{o b s}\left[c_{i-1}\right] / u u_{2}}\left|y_{i} \tau_{2}\right| .
$$

Suppose that $\left|x \tau_{2}\right|>\left|y \tau_{2}\right|+\left|c_{o b s}\left[c_{i-1}\right] \tau_{1}\right|$. Therefore:

$$
\left|c_{o b s}\left[c_{i-1}[c]\right] \tau_{1} \tau_{2} / u u_{1}\right|>\left|c_{o b s}\left[c_{i-1}[c]\right] \tau_{1} \tau_{2} / u u_{2}\right|,
$$

contradiction.

The second part of the proof is similar to that of Theorem 1.

\section{Conclusion}

We have presented an algorithm for computing a cover-context set which is a finite description of the often infinite set of observable contexts. We have shown that this computation applies to left-linear rewriting systems as well as an interesting class of non left-linear rewriting system, with any number of observable sorts. In the general case of a non left-linear system, we have proposed a procedure for computing a covercontext set. Once a cover-context set is computed, it is possible to use any first order theorem prover to prove observational properties, provided we use the context induction rule given below:

$$
\frac{\forall c\left[z_{s}\right] \in C C, \quad c\left[t_{1}\right]=c\left[t_{2}\right]}{t_{1}=t_{2}} \text { where } t_{1}, t_{2} \text { are terms of non observable sort. } s
$$

We plan to extend the computation of cover-context sets for a more general class of conditional specifications, and to use more refined observations based on operators or terms.

\section{Acknowledgements}

We would like to thank the referees for their valuable comments. 


\section{References}

[BBK94] G. Bernot, M. Bidoit, and T. Knapik. Behavioural approaches to algebraic specifications: a comparative study. Acta Informatica, 31(7):651-671, 1994.

[BBR98] N. Berregeb, A. Bouhoula, and M. Rusinowitch. Observational proofs with critical contexts. In Fundamental Approaches to Software Engineering, volume 1382 of Lecture Notes in Computer Science. Springer Verlag, 1998.

[BH93] B. Bauer and R. Hennicker. Proving the correctness of algebraic implementations by the isar system. In DISCO'93. Springer-Verlag, 1993.

[BH96] M. Bidoit and R. Hennicker. Behavioural theories and the proof of behavioural properties. Theoretical Computer Science, 165(1):3-55, 1996.

[DBG96] L. Dennis, A. Bundy, and I. Green. Using a generalisation critic to find bisimultations for coinductive proofs. In 14th Conference on Automated Deduction, volume 1249 of Lecture Notes in Computer Science, pages 276-290, 1996.

[GM99] J. Goguen and G. Malcolm. Hidden coinduction: Behavioral correctness proofs for objects. Mathematical Structures in Computer Science, 9(3):287-319, 1999.

[GM00] J. Goguen and G. Malcolm. A hidden agenda. Theoretical Computer Science, 245(1):55-101, 2000.

[Gut75] J. Guttag. The specification and application to programming of abstract data types. PhD thesis, University of Toronto, 1975.

[HB99] R. Hennicker and M. Bidoit. Observational logic. In Algebraic Methodology and Software Technology, volume 1548 of Lecture Notes in Computer Science, pages 263-277, 1999.

[Hen91] R. Hennicker. Context induction: a proof principle for behavioural abstractions and algebraic implementations. Formal Aspects of Computing, 3(4):3-55, 1991.

[JK89] J. Jouannaud and E. Kounalis. Automatic proofs by induction in theories without constructors. Information and Computation, 82:1-33, 1989.

[JR97] B. Jacobs and J. Rutten. A tutorial on coalgebras and coinduction. EATCS Bulletin, 62:222259, 1997.

[KC86] S. Kaplan and M. Choquer. On the decidability of quasi-reducibility. Bulletin of European Association for Theoretical Computer Science, 1986.

[KNZ86] D. Kapur, P. Narendran, and H. Zhang. On sufficient completeness and related properties of term rewriting systems. Acta Informatica, 24:395-415, 1986.

[Kou92] E. Kounalis. Testing for the ground (co-)reducibility property in term-rewriting systems. Theoretical Computer Science, 106:87-117, 1992. 
[MF98] M. Matsumoto and K. Futatsugi. Test set coinduction: Toward automated verification of behavioural properties. In 2nd International Workshop on Rewriting Logic and its Applications, Electronic Notes in Theoretical Computer Science. Elsevier Science, 1998.

[Pla85] D. Plaisted. Semantic confluence and completion method. Information and Control, 1985.

[Rei95] H. Reichel. An approach to object semantics based on terminal co-algebras. Mathematical Structures in Computer Science, 5:129-152, 1995.

[Sch92] O. Schoett. Two impossibly theorems on behavioural specifications. Acta Informatica, 29:595$621,1992$.

[SF95] K. Shmid and R. Fettig. Towards an efficient construction of test sets for deciding ground reducibility. In 6th Conference on Rewriting Techniques and Applications, volume 914 of Lecture Notes in Computer Science, 1995.

[Wir90] M. Wirsing. Algebraic specifications, chapter 13. MIT press, 1990. 
